\title{
Quantum supergroup structure of 1+1-dimensional quantum superplane, its dual and its differential calculus
}

\author{
M. EL Falaki* \\ Laboratoire de Physique Théorique, Faculté des Sciences, \\ Université Mohamed V, Av. Ibn Battota BP. 1014, Rabat, Morocco \\ Abdus Salam International Centre for Theoretical Physics, Trieste, Italy \\ E. H. Tahri ${ }^{\dagger}$ \\ Laboratoire de Physique Théorique, Faculté des Sciences, \\ Université Mohamed V, Av. Ibn Battota BP. 1014, Rabat, Morocco \\ Laboratoire de Physique Théorique et de Particules, Faculté des Sciences, \\ Université Mohammed I, BP. 524, 60000 Oujda, Moroccoł
}

October 1999

\begin{abstract}
We show that the $1+1$-dimensional quantum superplane introduced by Manin is a quantum supergroup according to the Faddeev-Reshetikhin-Takhtajan approach. We give its supermatrix element, its corresponding R-matrix and its Hopf structure. This new point of view allows us, first, to realize its dual Hopf superalgebra starting from postulated initial pairings. Second, we construct a right-invariant differential calculus on it and then deduce the corresponding quantum Lie superalgebra which as a commutation super-algebra appears classical, and as Hopf structure is a non-cocommutative q-deformed one. An isomorphism between the latter and the dual one obtained in the first method is given.
\end{abstract}

\footnotetext{
*E-mail:mantrach@fsr.ac.ma

†E-mail: tahrie@sciences.univ-oujda.ac.ma

†Permanent address
} 


\section{Introduction.}

In recent years there has been much interest in the concept of quantum groups and quantum algebras [1] [2] [3] [4]. Originating from investigations on the quantum inverse scattering methods and the Yang-Baxter equations, the quantum groups have found various applications in theoretical physics such as integrable fields theories, statistical models and conformal field theories in two-dimensions (see for example [5] [6] [7]).

Another major field starting in the seventies and still attracting a lot of attention among physicists and mathematicians is the field of super-symmetry. The extension of the activity on quantum groups to this field was started with the paper of Manin [8], where the multi-parametric quantum deformation of the supergroup $G L(m / n)$ was introduced. Since then intensive investigations concerning both structures and representations of the quantum supergroups were completed (see for example [9] [10] and references therein).

In Ref [8], Manin extended the notion of quantum space [11] to that of quantum super-space, called also quantum super-plane, of which the defining quadratic relations remain invariant under linear transformations. These endomorphisms constitute the quantum supergroup. From a rigorous mathematical point of view, the quantum superplane appears in this approach as a comodule over the corresponding quantum supergroup. The quantum (super)space has been then envisioned by many as a paradigm for the general program of quantum deformed physics. The most hopped for applications includes a possible role in a future quantized theory of gravity [12]. In this direction many efforts have been accomplished in order to develop its differential structure [13]. But all these constructions are made in the sense of [14 and not, as far as we know, in the sense of Woronowicz because of the lack of the Hopf structure of the quantum super-plane.

More recently, one of us 15 has introduced the quantum plane as a quantum group according to the Faddeev-Reshetikhin-Takhtajan approach [3]. In the present paper we extend the above investigation to the $1+1$-dimensional quantum super-plane. In this new approach the quantum super-plane appears as a quantum sub-supergroup of $S L_{q}(1 / 1)$. We give its super-matrix element, its corresponding R-matrix and its Hopf structure. The latter appears to be cocommutative. We then realize its dual Hopf superalgebra starting from postulated initial pairings. We also construct a right invariant differential calculus on it and we deduce its corresponding quantum Lie superalgebra which is as commutation superalgebra, up to a redefinition of the generators, the classical $N=1$ supersymmetric algebra, but its Hopf 
structure is a non-cocommutative $q$-deformed one. This quantum Lie superalgebra is isomorphic to the one we obtained by the above duality.

The paper is organized as follows. In section 2, we recall the Manin approach. In section 3 , the $1+1$-dimensional quantum superplane $K_{q}^{1 / 1}$ is made in the FRT approach, we also discuss some important properties of the quantum supermatrix considered to be a group element of $K_{q}^{1 / 1}$. In section 4, we find the dual Hopf superalgebra. In section 5 , we construct a right invariant differential calculus. In section 6, we derive the quantum Lie superalgebra, for which we give the Hopf structure in section 7 . Finally, we give some concluding remarks in section 8 .

\section{Review of the Manin approach}

The simplest example illustrating this approach is the one parameter quantum deformation $G L_{q}(1 / 1)$ of the supergroup $G L(1 / 1)$ of $2 \times 2$ supermatrices with two bosonic and two fermionic matrix elements. The construction of this quantum supergroup is well studied in [8] [16] [17]. It is generated by the elements of a quantum supermatrix:

$$
M=\left(\begin{array}{ll}
a & \beta \\
\gamma & d
\end{array}\right)
$$

which obey the following supercommutation relations:

$$
\begin{array}{ll}
a \beta=q \beta a, & d \beta=q \beta d \\
a \gamma=q \gamma a, & d \gamma=q \gamma d \\
\beta \gamma+\gamma \beta=0, & \beta^{2}=\gamma^{2}=0 \\
a d-d a=-\lambda \beta \gamma, &
\end{array}
$$

where $\lambda=q-q^{-1}$ and the generators $a$ and $d$ are even with parity $\hat{a}=\hat{d}=0$ and $\beta$, $\gamma$ are odd with parity $\hat{\beta}=\hat{\gamma}=1$ (called Grassmanian). The supermatrix $M$ defines the basic representation of $G L_{q}(1 / 1)$, i.e., $M$ is considered as a group element. Thus, in this sense, the quantum supergroup $G L_{q}(1 / 1)$ appears as a deformation of the algebra of polynomials function on $G L(1 / 1)$, the later is recovered when one takes $q \rightarrow 1$.

The relations (2) may be succinctly expressed in terms of the graded RTT equation as

$$
R M_{1} M_{2}=M_{2} M_{1} R
$$


where

$$
\begin{aligned}
\left(M_{1}\right)_{k l}^{i j} & =(M \otimes 1)_{k l}^{i j}=(-1)^{(\hat{k}(\hat{j}+\hat{l}))} M_{k}^{i} \delta_{l}^{j} \\
\left(M_{2}\right)_{k l}^{i j} & =(1 \otimes M)_{k l}^{i j}=(-1)^{(\hat{i}(\hat{k}+\hat{l}))} M_{l}^{j} \delta_{k}^{i}
\end{aligned}
$$

and the matrix $R$ is given by:

$$
R=\left(\begin{array}{cccc}
q & 0 & 0 & 0 \\
0 & 1 & 0 & 0 \\
0 & \lambda & 1 & 0 \\
0 & 0 & 0 & q^{-1}
\end{array}\right)
$$

which is a solution of the graded quantum Yang-Baxter equation:

$$
R_{12} R_{13} R_{23}=R_{23} R_{13} R_{12}
$$

arising from the associativity requirement of $G L_{q}(1 / 1)$.

In this formulation one can define the quantum superdeterminant:

$$
\begin{aligned}
\mathcal{D}=S \operatorname{det}(M) & =a d^{-1}-\beta d^{-1} \gamma d^{-1} \\
& =d^{-1} a-d^{-1} \beta \gamma d^{-1}
\end{aligned}
$$

provided $d^{-1}$ exists, and it can be checked that it is central, i.e., it commutes with all elements of $M$. Therefore, by imposing the relation $\mathcal{D}=1$, we may define a quantum deformation $S L_{q}(1 / 1)$ by analogy with the classical restriction to the special linear supergroup. The inverse supermatrix can be now defined. It is given by

$$
M^{-1}=\left(\begin{array}{cc}
a^{-1}+a^{-1} \beta d^{-1} \gamma a^{-1} & -a^{-1} \beta d^{-1} \\
-d^{-1} \gamma a^{-1} & d^{-1}+d^{-1} \gamma a^{-1} \beta d^{-1}
\end{array}\right) .
$$

So the Hopf structure of $G L_{q}(1 / 1)$ is given by the following:

$$
\begin{gathered}
\Delta(M)=M \dot{\otimes} M=\left(\begin{array}{rr}
a \otimes a+\beta \otimes \gamma & a \otimes \beta+\beta \otimes d \\
\gamma \otimes a+d \otimes \gamma & \gamma \otimes \beta+d \otimes d
\end{array}\right) \\
\varepsilon(M)=\left(\begin{array}{rr}
1 & 0 \\
0 & 1
\end{array}\right) \\
S(M)=M^{-1} .
\end{gathered}
$$

Now let us recall some statements related to the supermatrix $M$ considered as group element (or a point) of $G L_{q}(1 / 1)$. If one takes two copies $M$ and $M^{\prime}$ such that their elements pairwise supercommute, then the products $M M^{\prime}$ and $M^{\prime} M$ are group 
elements of $G L_{q}(1 / 1)$. Furthermore, the inverse $M^{-1}$ is actually a group element of $G L_{q^{-1}}(1 / 1)$ rather than $G L_{q}(1 / 1)$, and, in general, the n-th power of $M, n \in \mathbb{Z}$, is a group element of $G L_{q^{n}}(1 / 1)$.

Manin has defined in correspondence with the quantum supergroup what is called the quantum superplane or 'quantum superspace' and the dual of it. This quantum superplane or, rather, the polynomial function ring on it, denoted by $K_{q}^{1 / 1}$, is generated by two coordinates $x$ bosonic with parity $\hat{x}=0$ and $\theta$ fermionic with parity $\hat{\theta}=1$ such that:

$$
\begin{array}{ll}
x \theta & =q \theta x \\
\theta^{2}=0 . & (q \neq 0,1)
\end{array}
$$

The dual, denoted by ${ }^{*} K_{q}^{1 / 1}$, is generated by coordinates $\xi$ with parity 1 and $y$ with parity 0 and the following supercommutation relations:

$$
\begin{array}{ll}
\xi y=q^{-1} y \xi & (q \neq 0,1) \\
\xi^{2}=0 . &
\end{array}
$$

The quantum supergroup $G L_{q}(1 / 1)$ appears as endomorphisms of the quantum superplanes $K_{q}^{1 / 1}$ and ${ }^{*} K_{q}^{1 / 1}$ under which the quadratic relations (12) and (13) remain invariant. So Manin proved the equivalence between the defining quantum supergroup supercommutation relations (2) and the quadratic relations (12) and (13). In a more rigorous mathematical point of view, the quantum superplanes $K_{q}^{1 / 1}$ and ${ }^{*} K_{q}^{1 / 1}$ are viewed as comodules over $G L_{q}(1 / 1)$ via the following (left) coactions

$$
\begin{aligned}
& \delta(X)=M \dot{\otimes} X=\left(\begin{array}{c}
a \otimes x+\beta \otimes \theta \\
\gamma \otimes x+d \otimes \theta
\end{array}\right) \\
& \delta(\Theta)=M \dot{\otimes} \Theta=\left(\begin{array}{c}
a \otimes \xi+\beta \otimes y \\
\gamma \otimes \xi+d \otimes y
\end{array}\right)
\end{aligned}
$$

where $X$ and $\Theta$ are column vectors of components $x, \theta$ and $\xi, y$, respectively.

\section{Quantum superplane in new approach.}

Let us consider a particular copy of $G L_{q}(1 / 1)$ where $a=d=x$ and $\beta=\gamma=\theta$ that is:

$$
A=\left(\begin{array}{ll}
x & \theta \\
\theta & x
\end{array}\right),
$$


in this case the commutation relations (2) reduce to the quadratic relations (12) defining the quantum superplane $K_{q}^{1 / 1}$. Note that the superdeterminant of $A$ is equal to 1 , so it may be viewed also as a particular copy of $S L_{q}(1 / 1)$. Its quantum inverse supermatrix is given by

$$
A^{-1}=\left(\begin{array}{cc}
x^{-1} & -x^{-1} \theta x^{-1} \\
-x^{-1} \theta x^{-1} & x^{-1}
\end{array}\right) .
$$

This leads to our following main result.

\section{Thoerem.}

The quantum Manin superplane $K_{q}^{1 / 1}$ generated by the coordinates $x$ with parity 0 and $\theta$ with parity 1 and extended by the inverse $x^{-1}$ satisfying the quadratic relations $x \theta=q \theta x$ and $\theta^{2}=0$ is a quantum supergroup generated by elements of the supermatrix of type A modulo the graded RTT equation (3) where the matrix $R$ is given by (5). Its Hopf structure is given explicitly by:

$$
\begin{aligned}
& \Delta(x)=x \otimes x+\theta \otimes \theta, \\
& \Delta(\theta)=\theta \otimes x+x \otimes \theta, \\
& \varepsilon(x)=1, \\
& \varepsilon(\theta)=0, \\
& S(x)=x^{-1} \\
& S(\theta)=-x^{-1} \theta x^{-1} .
\end{aligned}
$$

We see that it is a cocommutative Hopf superalgebra.

There are curious properties applicable to this quantum supermatrix considered as a group element of $K_{q}^{1 / 1}$. They assert that:

a) If $A$ and $A^{\prime}$ are two copies of $K_{q}^{1 / 1}$ such that their entries pairwise supercommute, then the products $A A^{\prime}$ and $A^{\prime} A$ are group elements of $K_{q}^{1 / 1}$.

b) the n-th power of $A$ is a group element of $K_{q^{n}}^{1 / 1}$ given by:

$$
A^{n}=\left(\begin{array}{cc}
x^{n} & {[n]_{q} \theta x^{n-1}} \\
{[n]_{q} \theta x^{n-1}} & x^{n}
\end{array}\right),
$$

where $[n]_{q}=\left(1-q^{n}\right) /(1-q)$. Note that if we take $n=-1$ we find the inverse (17) (since $\left.[-1]_{q}=-q^{-1}\right)$. Furthermore, if there exists an integer $n$ such that $q^{n}=1$, then the n-th power of $A$ (19) reduces to

$$
A^{n}=\left(\begin{array}{cc}
x^{n} & 0 \\
0 & x^{n}
\end{array}\right)
$$


which gives a nilpotent supermatrix when $x^{n}=0$ or unit supermatrix when $x^{n}=1$.

So in this sense, the quantum superplane $K_{q}^{1 / 1}$ can be considered as a quantum subsupergroup of $S L_{q}(1 / 1)$.

\section{The dual of $K_{q}^{1 / 1}$}

We will apply here the method introduced by Sudbery in [18], where he obtained $\mathcal{U}_{q}(\operatorname{sl}(2)) \otimes \mathcal{U}(u(2))$ as the algebra of tangent vectors at the identity, and generalized to more complicated algebras by Dobrev [19. The application of this method to the multiparameter supergroup $G L_{u \mathbf{q}}(\mathrm{m} / n)$ is given in [9]. It consists of using the duality between (super)bialgebras or Hopf (super)algebras to obtain the unknown dual of a known (super)algebra.

Two bialgebras $\mathcal{U}$ and $\mathcal{A}$ are said to be in duality [20] if there exists a doubly nondegenerate bilinear form

$$
\langle,\rangle: \mathcal{U} \times \mathcal{A} \longrightarrow \mathbb{C} \quad(u, a) \longmapsto\langle u, a\rangle \quad u \in \mathcal{U}, a \in \mathcal{A}
$$

such that, for $u, v \in \mathcal{U}, a, b \in \mathcal{A}$

$$
\begin{aligned}
\langle u, a b\rangle & =\left\langle\delta_{\mathcal{U}}(u), a \otimes b\right\rangle \\
\langle u v, a\rangle & =\left\langle u \otimes v, \delta_{\mathcal{A}}(a)\right\rangle \\
\left\langle u, 1_{\mathcal{A}}\right\rangle & =\varepsilon_{\mathcal{U}}(u), \quad\left\langle 1_{\mathcal{U}}, a\right\rangle=\varepsilon_{\mathcal{A}}(a) .
\end{aligned}
$$

For dual Hopf algebras one adds

$$
\left\langle\gamma_{\mathcal{U}}(u), a\right\rangle=\left\langle u, \gamma_{\mathcal{A}}(a)\right\rangle
$$

To extend this to superalgebras or Hopf superalgebras, we take into account that the tensor product is also graded, and, if (using Sweedler's notation) $\delta_{\mathcal{U}}=\sum u_{(1)} \otimes$ $u_{(2)}, \delta_{\mathcal{A}}=\sum a_{(1)} \otimes a_{(2)}$, then

$$
\begin{aligned}
& \langle u, a b\rangle=\sum(-1)^{\widehat{u_{(2)}}}\left\langle u_{(1)}, a\right\rangle\left\langle u_{(2)}, b\right\rangle, \\
& \langle u v, a\rangle=\sum(-1)^{\widehat{v a(1)}}\left\langle u, a_{(1)}\right\rangle\left\langle v, a_{(2)}\right\rangle .
\end{aligned}
$$

It is enough to define the pairing (21) between the generating elements of the two algebras. The pairing may then be extended to all elements of $\mathcal{U}$ and $\mathcal{A}$ by using relations (22). 
To find the unknown superalgebra, it is enough to give the pairing between the generating elements of the unknown superalgebra with an arbitrary element of the PBW basis of the known superalgebra. So, we first need to fix a PBW basis of $K_{q}^{1 / 1}$. This basis consists of monomials

$$
h=x^{m} \theta^{n}
$$

where $m \in \mathbb{Z}$ and $n \in\{0,1\}$. Let us then denote the dual superalgebra by $\mathcal{U}_{q}(k(1 / 1))$ and its generating elements by $\chi$ and $\phi$. Following [19] we shall postulate the pairing $\langle Z, h\rangle Z=\chi, \phi, h$ from (25), as we use the classical tangent vector at the identity :

$$
\begin{aligned}
& \langle\chi, h\rangle=m \delta_{n, 0}, \\
& \langle\phi, h\rangle=\delta_{n, 1} .
\end{aligned}
$$

We also note that, from the duality properties, it follows

$$
\left\langle 1_{\mathcal{U}}, h\right\rangle=\varepsilon(h)=\delta_{n, 0} .
$$

To obtain the commutation relations between the generators $\chi$ and $\phi$, we first need to evaluate the action of their bilinear product on the elements of $K_{q}^{1 / 1}$. Using the defining relations we obtain

$$
\begin{aligned}
& \langle\chi \phi, h\rangle=(m+1) \delta_{n, 0}, \\
& \langle\phi \chi, h\rangle=(m+1) \delta_{n, 1}, \\
& \left\langle(\phi)^{2}, h\right\rangle=-\frac{1-q^{-2 m}}{1-q^{-2}} \delta_{n, 0} .
\end{aligned}
$$

Thus we have the commutation relations :

$$
\begin{aligned}
& \chi \phi-\phi \chi=0, \\
& (\phi)^{2}=-\frac{1-q^{-2 \chi}}{1-q^{-2}} .
\end{aligned}
$$

The Hopf structure of this superalgebra may be deduced by using the duality. We start with the coproduct, i.e., we shall use the relations

$$
\langle Z, h\rangle=\left\langle\delta_{\mathcal{U}}(Z), h_{1} \otimes h_{2}\right\rangle
$$

for every splitting $h=h_{1} h_{2}$. Thus we get

$$
\begin{aligned}
& \delta_{\mathcal{U}}(\chi)=\chi \otimes 1+1 \otimes \chi \\
& \delta_{\mathcal{U}}(\phi)=\phi \otimes q^{-\chi}+1 \otimes \phi .
\end{aligned}
$$


the counit relations in $\mathcal{U}_{q}(k(1 / 1))$ are given by

$$
\varepsilon_{\mathcal{U}}(Z)=0, \quad Z=\chi, \phi
$$

which follows easily from

$$
\left\langle Z, 1_{\mathcal{A}}\right\rangle=0=\varepsilon_{\mathcal{U}}(Z) .
$$

Finally, the antipode map of $\mathcal{U}_{q}(k(1 / 1))$ follows from

$$
\left\langle\gamma_{\mathcal{U}}(Z), h\right\rangle=\left\langle Z, \gamma_{\mathcal{A}}(h)\right\rangle \text {. }
$$

It is given by

$$
\begin{aligned}
& \gamma_{\mathcal{U}}(\chi)=-\chi, \\
& \gamma_{\mathcal{U}}(\phi)=-q^{\chi} \phi .
\end{aligned}
$$

Now let us make the following redefinition:

$$
\phi^{\prime}=i q^{(\chi-1) / 2} \phi, \quad i^{2}=-1
$$

then we get the following quantum deformed enveloping superalgebra (dropping the primes)

$$
\begin{aligned}
& \chi \phi-\phi \chi=0 \\
& (\phi)^{2}=[\chi]
\end{aligned}
$$

where $[x]=\left(q^{x}-q^{-x}\right) /\left(q-q^{-1}\right)$. Its Hopf structure is now given by:

$$
\begin{aligned}
& \delta_{\mathcal{U}}(\chi)=\chi \otimes 1+1 \otimes \chi, \\
& \delta_{\mathcal{U}}(\phi)=\phi \otimes q^{-\chi / 2}+q^{\chi / 2} \otimes \phi . \\
& \varepsilon_{\mathcal{U}}(\chi)=\varepsilon_{\mathcal{U}}(\phi)=0, \\
& \gamma_{\mathcal{U}}(\chi)=-\chi, \quad \gamma_{\mathcal{U}}(\phi)=-\phi .
\end{aligned}
$$

\section{Differential calculus on $K_{q}^{1 / 1}$}

The differential calculus on quantum group was initiated by Woronowicz [21] [22]. Here we follow the same techniques used in 23] to construct the right invariant calculus on the two-parameter deformation of $G L(2)$ and extended in [24] and [25] to the one and two-parameter deformation of $G L(1 / 1)$, respectively. 
We introduce at first the exterior differential $d$ which satisfies the nilpotency and the graded Leibniz rule,

$$
\begin{aligned}
& d^{2}=0, \\
& d(f g)=(d f) g+(-1)^{\hat{f}} f(d g),
\end{aligned}
$$

where $f, g$ are functions of the variables $x, \theta$ and $\hat{f}$ is the parity of $f$. In analogy with the classical differential geometry, one can construct from $A$ and the one-forms $d A$, via the matrix valued one-forms $\Omega$, the right-invariant Cartan-Maurer forms as

$$
\Omega=(d A) A^{-1}=\left(\begin{array}{cc}
\omega & v \\
v & \omega
\end{array}\right) .
$$

Writing this as $d A=\Omega A$, one can express the basic one-forms $d x$ and $d \theta$ in terms of Cartan-Maurer forms as

$$
\begin{aligned}
& d x=\omega x+v \theta \\
& d \theta=v x+\omega \theta .
\end{aligned}
$$

The exterior differential $d$ can then be written as

$$
\begin{aligned}
d & =d x \partial_{x}+d \theta \partial_{\theta} \\
& =\omega D_{x}+v D_{\theta} .
\end{aligned}
$$

Using the nilpotency of the differential $d$ and (41) it follows that:

$$
\begin{aligned}
& d \omega=\omega^{2}-v^{2} \\
& d v=\omega v-v \omega
\end{aligned}
$$

which we call the Cartan-Maurer equations.

Now we want to calculate the $q$-deformed supercommutation relations between the basic variables $x, \theta$ and the Cartan-Maurer forms. Using a similar restriction as in [24] due to the right-invariance of the Cartan-Maurer forms, we suggest

$$
\begin{aligned}
& x v=q v x, \quad \theta v=q v \theta, \\
& x \omega=F \omega x, \quad \theta \omega=-F \omega \theta,
\end{aligned}
$$

where $F$ is an arbitrary complex number. Then the action of $d$ on the quadratic relations (12) do not lead to any inconsistency, while the consistence of $d$ with (44) requires the following:

$$
\begin{aligned}
& \omega v=v \omega \\
& \omega^{2}=k v^{2}, \quad k=\frac{q^{2}-F}{F(F+1)} .
\end{aligned}
$$


Using this, the Cartan-Maurer equations (43) reduce to

$$
\begin{aligned}
& d \omega=(k-1) v^{2} \\
& d v=0
\end{aligned}
$$

\section{Quantum Lie superalgebra}

Now let us return to the expression of $d$ in (42), the elements $D_{x}$ and $D_{\theta}$ generate the quantum Lie superalgebra associated to the quantum superplane $K_{q}^{1 / 1}$. To calculate the supercommutation relations between these generators we use the nilpotency of $d$

$$
\begin{aligned}
0=d^{2}(f) & =d\left(\omega D_{x} f+v D_{\theta} f\right) \\
& =(d \omega) D_{x} f+(d v) D_{\theta} f-\omega\left(\omega D_{x}+v D_{\theta}\right) D_{x} f+ \\
& +v\left(\omega D_{x}+v D_{\theta}\right) D_{\theta} f
\end{aligned}
$$

where $f$ is an arbitrary function of the variables $x, \theta$. Then, using (45) and (46), it follows that

$$
\begin{aligned}
& D_{x} D_{\theta}-D_{\theta} D_{x}=0 \\
& D_{\theta}^{2}=k D_{x}^{2}+(1-k) D_{x} .
\end{aligned}
$$

We will denote this superalgebra by $k_{q, F}(1 / 1)$. Note that if we make the following change:

$$
T_{x}=k D_{x}^{2}+(1-k) D_{x}
$$

then we get

$$
\begin{aligned}
& T_{x} D_{\theta}-D_{\theta} T_{x}=0, \\
& D_{\theta}^{2}=T_{x},
\end{aligned}
$$

which are the commutation relation of the 'classical' $N=1$ supersymmetric algebra. So the quantum deformation does not affect the Lie superalgebra structure.

Now it is possible to calculate the supercommutation relations between the generators of $k_{q, F}(1 / 1)$ and the basic coordinates. To this end, we use the graded Leibniz rule by comparing the coefficients of the Cartan-Maurer forms when acting by $d$ on $x f$ or $\theta f$. Then we obtain

$$
\begin{array}{ll}
D_{x} x=x+F x D_{x}, & D_{x} \theta=\theta+F \theta D_{x} \\
D_{\theta} x=\theta+q x D_{\theta}, & D_{\theta} \theta=x-q \theta D_{\theta} .
\end{array}
$$


On the other hand, if we multiply the second relation in (48) from the right by $x$ or $\theta$ and then pull it to the left using the commutation relations (51), then we get

$$
D_{\theta}^{2}-\frac{k F^{2}}{q^{2}} D_{x}^{2}-\frac{F(1+k)}{q^{2}} D_{x}=0
$$

from which it follows that $F$ must be equal to $q^{2}$ or $q$. We will see in the next section that these two solutions are equivalent.

\section{Hopf structure of the superalgebra $k_{q, F}(1 / 1)$}

Now we shall use the graded Leibniz rule for arbitrary functions $f$ and $g$ of coordinates $x, \theta$ to derive the Hopf structure of $k_{q, F}(1 / 1)$. An arbitrary function is in fact a linear combination of monomials in the basis (25).

We first need to calculate the commutation relations between the Cartan-Maurer one-forms and functions. From (44) we have

$$
\begin{aligned}
& f \omega=(-1)^{\hat{f}} w F^{N} f \\
& f v=v q^{N} f .
\end{aligned}
$$

where $N$ is an operator number acting on monomial as

$$
N\left(x^{m} \theta^{n}\right)=(m+n) x^{m} \theta^{n}
$$

and may be related to the operator $D_{x}$. To see this we calculate the action of $D_{x}$ on functions which may be achieved by calculating its commutation relation with $h$. Indeed we have

$$
D_{x} h=\frac{1-F^{m+n}}{1-F} h+F^{n} h D_{x}
$$

from which we get

$$
D_{x}=\frac{1-F^{N}}{1-F}
$$

Therefore, using the graded Leibniz rule applied to the product of functions $f$ and $g$, and the expression of $d$ in terms of $D_{x}$ and $D_{\theta}$ (42) with the help of (53), this leads to the following:

$$
\begin{aligned}
& D_{x}(f g)=D_{x}(f) g+F^{N} f D_{x}(g), \\
& D_{\theta}(f g)=D_{\theta}(f) g+(-1)^{\hat{f}} q^{N} f D_{\theta}(g),
\end{aligned}
$$


from which we get the coproduct:

$$
\begin{aligned}
& \delta\left(D_{x}\right)=D_{x} \otimes 1+F^{N} \otimes D_{x} \\
& \delta\left(D_{\theta}\right)=D_{\theta} \otimes 1+q^{N} \otimes D_{\theta}
\end{aligned}
$$

where use was made of the graded tensor product: $(a \otimes b)(f \otimes g)=(-1)^{\hat{b} \hat{f}} a(f) \otimes b(g)$, $a, b \in k_{q, F}(1 / 1)$ and $f, g \in K_{q}^{1 / 1}$. The counit and antipode relations in $k_{q, F}(1 / 1)$ may be calculated by using the basic axioms of Hopf superalgebras [20] [8]:

$$
\begin{aligned}
& \left(\varepsilon_{k} \otimes i d\right) \delta_{k}(u)=u, \\
& m\left(i d \otimes \gamma_{k}\right) \delta_{k}(u)=\varepsilon_{k}(u),
\end{aligned}
$$

where $u \in k_{q, F}(1 / 1)$ and $m$ is the multiplication. So we obtain

$$
\begin{aligned}
& \varepsilon_{k}\left(D_{x}\right)=\varepsilon_{k}\left(D_{\theta}\right)=0 \\
& \gamma_{k}\left(D_{x}\right)=-F^{-N} D_{x}, \quad \gamma_{k}\left(D_{\theta}\right)=-q^{-N} D_{\theta} .
\end{aligned}
$$

Thus, we have a $q$-deformed non-cocommutative Hopf structure depending on the parameter $q$. Furthermore, we note that the two forms of (58), and (60) according to the two solutions of $F=q^{2}$ or $q$ are consistent with the redefinition (49). Therefore, we may view the two solutions of $F$ as corresponding to changes of basis for the quantum enveloping superalgebra and are equivalent as such. So, it is convenient to choose the solution $F=q^{2}$ which is the simplest one. We can then transform this superalgebra to the form obtained in Section 4 (c.f., (37) (38)) by making the following changes:

$$
\begin{aligned}
& D_{x} \equiv \frac{1-q^{2} \chi}{1-q^{2}} \\
& D_{\theta} \equiv q^{(\chi-1) / 2} \phi
\end{aligned}
$$

which are consistent with the commutation relations and the Hopf structure.

\section{Concluding remarks}

We have shown that the 1+1-dimensional quantum superplane, which is well known in the literature as comodule over its corresponding quantum supergroup, may be incorporated in the Faddeev-Reshetikhin-Takhtajan approach to quantum (super)groups. We have given its supermatrix element, its corresponding R-matrix and its Hopf structure which is a cocommutative one. We then derived its dual 
Hopf superalgebra starting from a postulated initial pairing. We also constructed a right differential calculus on it and we deduced the corresponding quantum Lie superalgebra. As a commutation superalgebra, the latter coincides with the classical $\mathrm{N}=1$ supersymmetric algebra, but its Hopf structure is a non-cocommutative $q$-deformed one. We also prove that there is an isomorphism between this quantum Lie superalgebra and the one we obtained using postulated duality. To finish let us stress that the concept of $q$-deformation of Lie (super)group and (super)space is physically consistent when it is performed in the context of Hopf (super)algebra. So we expect that this new approach to the quantum superplane and the differential calculus introduced on it here will be a starting point to construct more consistent $q$-deformed physical models.

Acknowledgments. One of the authors MEF is grateful for hospitality at the Abdus Salam ICTP, where part of this work was achieved, to Prof. R. Floreanini, and to Prof. S. Randjbar-Daemi for reading this paper.

\section{References}

[1] V. G. Drinfeld, Quantum groups, Proc. Int. Congress of Math., Berkely, CA 1986 (AMS, Providence, RI 1987) V1 798.

[2] M. Jimbo, Lett. Math. Phys. 10 (1985) 63; ibid 11 (1986) 247.

[3] L. D. Faddeev, N. Yu. Reshetikhin and L. Takhtajan, Alg. Anal. 1 (1987) 178.

[4] S. L. Woronovicz, Comm. Math. Phys. 111 (1987) 613.

[5] H. -D. Doebner, J. -D. Henning (eds), Quantum groups, Lect. notes Phys. V370, Springer 1990.

[6] T. Curtright, D. Fairlie and C. Zachos (eds), Quantum groups, Proceeding of the Aragonne Workshop, World Scientific 1990.

[7] J. Lukierski, Z. Popowicz and J. Sobczyk (eds), Quantum groups: Formalism and applications, XXX Karpacz Winter school of theoretical physics, Polish Scientific Publishers PWN 1995.

[8] Yu. I. Manin, Comm. Math. Phys. 123 (1989) 163. 
[9] V. K. Dobrev and E. H. Tahri, Int. J. Mod. Phys A13 (1998) 4339.

[10] N. A. Ky, Superalgebras, their quantum deformations and the induced representation method, math. QA/9810170.

[11] Yu. I. Manin, "Quantum groups and non-commutative geometry ", Preprint Montreal Univ. CRM-1561, 1988.

[12] S. Majid, Fondation of quantum group theory, Cambridge Univ. Press 1995.

[13] T. Kobayashi and T. Ueamatsu, Z. Phys. C 56 (1992)193.

[14] J. Wess and B. Zumino, Nucl Phys. (Proc. Suppl.) B18 (1990) 302.

[15] E. H. Tahri, J. Math. Phys, 39 5, (1998) 2983.

[16] J. Schwenk, W. B. Schmidke and S. Vokos, Z. Phys. C 46 (1990) 643.

[17] E. Corrigan, D. B. Fairlie, P. Fletcher and R. Sasaki, J. Math. Phys. 31(4) (1990) 776.

[18] A. Sudbery, Proceeding of the Workshop on Quantum groups, Aragone National Lab. (1990), Eds. T. Curtright, D. Fairlie and C. Zachos (World Sci, 1990) pp 33 .

[19] V. K. Dobrev, J. Math. Phys. 33 (1992) 3419.

[20] E. Abe, Hopf Algebras, Cambridge Tracts in Math. N74 (Cambridge Univ. Press, 1980).

[21] S. L. Woronowicz, Publ. Res. Inst. Math. Sci. 23 (1987) 117.

[22] S. L. Woronowicz, Comm. Math. Phys. 122 (1989) 125.

[23] A. Schirmacher, J. Wess and B. Zumino, Z. Phys. C 49 (1991) 317.

[24] W. B. Schmidke, S. P. Vokos and B. Zumino, Z. Phys. C 48 (1990) 249.

[25] C. Burdik and R. Tomasek, Lett. Math. Phys. 26 (1992) 97. 